\title{
Die Zeit und das Geld
}

\author{
Heinz-Michael Just
}

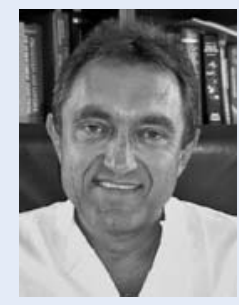

Heinz-Michael Just



er Slogan Zeit ist Geld hat längst Einzug in die Medizin gehalten. Dabei ist Zeit das Wichtigste, was ein Patient braucht: Zeit des Arztes, ihm zuzuhören, Zeit der Pflegekräfte, ihn zu pflegen und Zeit für ihn selbst, gesund zu werden. Nichts davon ist heute mehr vorhanden. Der Arzt hat am liebsten nur die „Werte“, die Pflegenden sind mit dokumentieren beschäftigt und der Patient „muss raus“, weil sonst nicht genug Gewinn übrig bleibt. Der Patient ist freilich immer noch Mittelpunkt, nur anscheinend unter veränderten Vorzeichen: war er früher der, dem man bestrebt war, möglichst optimale Hilfe zuteil werden zu lassen, ist er heute die „Handelsware", die es gilt, betriebswirtschaftlich maximal auszubeuten. Dass er dabei Gefahr läuft, auf dem Papier noch kränker zu erscheinen, als er ohnehin ist und dass bei ihm überflüssige diagnostische und therapeutische Prozeduren durchgeführt werden, gilt inzwischen als akzeptierter Kollateralschaden - das System will es so. Nicht mehr die Erkrankung scheint zu bestimmen, was mit einem Patienten geschieht, sondern die möglichen DRGs, die man über ihn abrechnen kann.

Die Kostensteigerungen der letzten Jahre sind zum erheblichen Teil diesem System geschuldet. Erst machte man Kliniken zu „Profitzentren“ was Konkurrenzdenken hervorrief. Dass daraufhin jede noch so kleine Klinik ihren Patienten eine möglichst umfassende Diagnostik und Therapie anbieten wollte, ist nur logisch. Die Folge dürften in erheblichem Umfang kostenträchtige „Amortisationsindikationen“ gewesen sein, denn die teuren Geräte mussten sich betriebswirtschaftlich rechnen. Oder vermeidbare oftmals infektionsbedingte Komplikationen, weil bei bestimmten Eingriffen aufgrund zu geringer Fallzahlen die Erfahrung fehlte. Dann folgten Chefarztverträge mit Zielvereinbarungen nach deren Bekanntwerden in der Öffentlichkeit irritiert die Frage gestellt wurde, ob dadurch vielleicht Indikationsstellungen im Einzelfall beeinflusst werden könnten: Statt einer konservativen Therapie ein operativer Eingriff mit allen damit verbundenen Risiken, incl. nosokomialer Infektion.

Dass nosokomiale Infektionen nicht nur durch die in den Medien so beliebten „Killerkeime“ entstehen, sondern überwiegend durch „normale“ Bakterien von
Haut- oder Schleimhaut der Ärzte und Pfleger, meist aber des Patienten selbst, dringt nicht so recht ins Bewusstsein. Stattdessen beschuldigt man gerne Bakterien aus der Umgebung: Wasserkeime, gegen die die Industrie Wasserfilter anbietet, oder auf Flächen nachweisbare Bakterien, die durchaus von den beteiligten Personen stammen. Dafür bietet die Industrie viel Antimikrobielles: imprägnierte Stoffe, beschichtete Oberflächen (seien es Arbeitsflächen oder Wandfliesen), Kupfergegenstände wie Türgriffe, Lichtschalter und Handläufe, und wer es ganz gründlich plant, baut gleich mit antimikrobiell wirksamen Bausteinen. Diese Sicht von Hygiene eröffnet der Industrie einen Milliardenmarkt und den Beschäftigten im Gesundheitswesen die Möglichkeit, keine eigene Betroffenheit zulassen zu müssen, denn es sind ja nicht sie, sondern es ist die Umgebung. Bis auf die Hände, die lassen sich schlecht wegretuschieren. Dafür gibt es alle Jahre wieder den europäischen „Händehygienetag“, mancherorts mit Bandenwerbung wie im Stadion und Ständen wie auf dem Jahrmarkt. Dass dadurch die Compliance der Beschäftigten im Gesundheitswesen deutlich besser geworden wäre, hat noch niemand in großem Stil zeigen können. Das daran Denken im täglichen Handeln ist das Entscheidende!

Um das zu fördern hat der Gesetzgeber 2011 Hygienemitarbeiter sowohl im ärztlichen als auch im pflegerischen Bereich verordnet [1] und 2013 eine Art Anschubfinanzierung nachgereicht [2]. Im Gesetz zur Beseitigung sozialer Überforderung bei Beitragsschulden in der Krankenversicherung wird beschrieben, unter welchen Voraussetzungen medizinische Einrichtungen wie viel Geld zur Einstellung, Aus- und Weiterbildung ihrer Hygienemitarbeiter bekommen. In einer Erläuterung wurde hierzu ausgeführt, dass diese Beträge mit den Versicherungen im Rahmen der DRG-Verhandlungen als Aufschlag auszuhandeln seien. Man kann dies durchaus als Ironie empfinden.

Dass sich dadurch die Grundeinstellung insbesondere mancher Ärzte ändern wird, die sich darin äußert, dass z.B. so grundlegende Forderungen wie die nach adäquater Händehygiene in der Praxis aus Zeitgründen als nicht durchführbar bezeichnet wird, ist nicht zu erwarten. Die zunehmende Zahl an Controllern im 
Verwaltungsbereich mag das Budget transparenter werden lassen, belastet es aber auch. Die zunehmende Zahl an Hygienemitarbeitern bietet dagegen eine Voraussetzung, notwendige Verhaltensweisen im Umgang mit Patienten zur Routine werden zu lassen, nosokomiale Infektionen (und damit Kosten) zu vermeiden. Allerdings nur, wenn begleitend dazu eine ausreichende Zahl an Ärzten und Pflegekräften die erforderliche Zeit für ihre verantwortungsvolle, Konzentration erfordernde Tätigkeit haben $[3,4]$ !

\section{Literatur}

1 Gesetz zur Verhütung und Bekämpfung von Infektionskrankheiten beim Menschen (Infektionsschutzgesetz - IfSG). http://www.gesetze-im-internet.de/bundesrecht/ifsg/ gesamt.pdf

2 Gesetz zur Beseitigung sozialer Überforderung bei Beitragsschulden in der Krankenversicherung. Bundesgesetzbl. Teil 1 Nr. 38 2013: $2423-2430$

3 Clements $\mathrm{A}$, Halton $\mathrm{K}$, Graves $\mathrm{N}$ et al. Overcrowding and understaffing in modern health-care systems: key determinants in meticillin-resistant Staphylococcus aureus transmission. Lancet Infect Dis 2008; 8: 427-434

4 Pittet D, Hugonnet S, Harbarth $\mathrm{S}$ et al. Effectiveness of a hospital-wide programme to improve compliance with hand hygiene. Lancet 2000; 356: 1307-1312 